\title{
CONCEPTION OF A NEW GENERATION OF 3D BIOMIMETIC MICROVALVES FOR MEDICAL APPLICATION
}

\author{
N. Labdelli ${ }^{*}$, S. Soulimane ${ }^{2}$ \\ ${ }^{1}$ Biomedical engineering research laboratory, Faculty of Technology Department of Biomedical \\ Engineering, University of Tlemcen, 13000, Algeria. \\ e-mail: nassima.labdelli@univ-tlemcen.dz \\ ${ }^{2}$ Biomedical engineering research laboratory, Faculty of Technology Department of Biomedical \\ Engineering, University of Tlemcen, 13000, Algeria. \\ e-mail: Soulimane.Sofiane@univ-tlemcen.dz \\ *corresponding author
}

\begin{abstract}
This paper reviews the development of a new microvalve with a cone-shaped tube, inspired by venous valves in the human body. Our microvalves allow fluid flow in one direction while restricting the flow in the opposite direction. When a microvalve is used to control the amount of drug delivery, the efficiency between inlet and outlet flow rate is the key control parameter for regulating and controlling the micro channel (opening/closing). This paper is devoted to the numerical study of flow rate changes in different microvalve geometries (3D) using a Fluid Structure Interaction (FSI) method with an Arbitrary Lagrangian Eulerian (ALE) approach. Numerical simulations were carried out in comsol Multiphysics. In addition, the macrovalve performance was analysed for several pressures where the effect of different geometrical parameters such as the length of the anchor, the diameter at the base and the angle of the cone were studied. An efficiency parameter $E_{f f}$ was employed to compare the different structures. For the best design obtained, it was found that the cone angle was the parameter having the most effect on the microvalves' characteristics, and the forward flow rate was more than doubled compared to the reverse leakage rate.
\end{abstract}

Keywords: Fluid structure interaction, Arbitrary Lagrangian Eulerian, COMSOL Multiphysics, Flow rate, Efficiency.

\section{Introduction}

Microvalves and micropumps are considered to be key functional components of microfluidic systems. Their performance directly affects the precision and reliability of the Micro Total Analysis System ( $\mu$ TAS) (Nordina et al. 2017). In addition, a micropump plays an important role in targeting transport of tracing substances (Laser et al. 2004, Woias et al. 2005). It can carry small and precise volume of liquids for chemical, biological or medical systems (Amrani et al. 2018). Micropumps are appropriate tools for drug delivery in the body, suitable for modern biotechnology drug, provideing high precision delivery (Cui et al. 2006, McAllister et al. 2000). Micropumps can be used for drug delivery with the desired amount of medicines at the 
appropriate time, which improves the therapeutic effect. With an increasing interest in the development of biochemical microsystems (Wenpeng et al. 2017), there have been numerous reports on the design and fabrication of a variety of micropumps (Partha and Toufique, 2017). These micropumps have recently emerged as a critical area of research because of their extensive potential applications in the fields of chemical analysis (Zolotov. 2020), biological instruments (Nickolay et al. 2004), and medical treatment (Wang et al. 2014). Various types of microvalves have been extensively developed and demonstrated (Victor et al. 2014, Liang-Yin and Wei Wang, 2017, Kwang and Chong 2006). They are classified into active (with actuation) and passive (without actuation) microvalves (Jin-yuan et al. 2020). Most conventional active microvalves couple a flexible diaphragm to an actuator capable of deflecting the diaphragm (Aleksandr 2017, Saggere 2015, Huang 2020). These actuators typically require high power consumption and the scaling of the resultant forces with respect to the size of the device is often unfavourable (Nabel. 2016), as well as complexity in their fabrication process. Thermally actuated microvalves are an example of active valves, their use, such as thermopneumatic (Yang et al. 2019), shape memory alloy (Shrikan et al. 2019, Ramchandani et al. 2017), and bimetallic (Srinivasa 2020, Lefevre et al. 2012), but the actuation mechanism is typically limited to temperature control. Passive microvalves are broadly classified as mechanical (valve-based) and non-mechanical (valveless). The mechanical pump has moving parts such as diaphragm/membrane and check valves, which control the fluid flow. On the other hand, non-mechanical micropump has no moving parts and the fluid flow requires conversion of non-mechanical energy into kinetic energy to supply the fluid with momentum. This is done by using a diffuser/nozzle arrangement in the micropump for fluid flow regulation. There are various special tubes in valveless micropumps (Barkat et al. 2018), such as diffuser/nozzles (Marwan et al. 2019), Tesla tubes (Zhuo et al. 2011), and sawtooth tubes (Xu et al. 2014). On the other side, the structures of the valveless micropumps are relatively simple comparing to the check valve micropumps and, thus, the fabrication processes of the valveless micropumps are less complicated. However, the valveless micropumps have inherent issues such as backflow and random flow. These issues may bring difficulties in precisely controlling pumping rates in applications. The previous research (Kwang and Chong 2006, He et al. 2015) indicates that the efficiency of the valveless micropumps with diffuser/nozzle elements is determined by the parameters (diverging angles and excitation frequency) (He et al. 2015). In contrast to valveless diaphragm micropumps, check valve diaphragm micropumps (Kang and Auner 2011, Kang et al. 2008) generally have better performance, providing a much wider linearly controllable pumping rate range and a higher pumping pressure; therefore, check valve micropumps are more attractive in microfluidic applications.

In this paper, we report on a new design of biomimetic passive microvalves inspired by natural venous valves. Our proposed structure, as shown in Fig.1, is useful to control the flow rate under certain pressure difference. Up to now, only few papers that discussed about micropumps with cone-shaped tubes have been found in our retrieved data, and their research is superficial (Zhang et al. 2017). Hence, static venous valves are surely more complicated than cone-shaped tubes whether in parallel forms or in series forms. In the same way, Yu et al. (2000, 2001) designed and fabricated soft valves based on a bi-strip stimuli-sensitive hydrogel that combine the actuation mechanism of bimetallic valves and the directional flow control of a check valve. However, they are limited by the nature of the fluid because their hydrogel valve not only operates as passive check valves, but also activates or deactivates autonomously based on the chemical environment of the fluid medium.

By combining the cone-shaped reported previously, we were able to simulate a 3D generic biomimetic soft check valve that mimics biological systems both structurally and functionally, based on the pressure gradient applied on different fluids. 
In this paper, firstly, we describe in detail the microvalve design using COMSOL Multiphysics software. Next, we study the influential parameters on the performance of our novel structure. Finally, we discuss the obtained results.

\section{Micropump structure design and simulation}

\subsection{Structure design}

We designed and modelled a three-dimensional soft microvalve based on a cone-shaped structure embedded in the bottom of the micro channel and controls the flow without any actuators. Figure 1 illustrates the structure of our microvalves. The idea is to make the microvalves as streamlined as possible for one direction and resembling a resistant body in the other direction. Geometric parameters have a direct influence on the performance of the microvalve. We can identify the following parameters: diameter at the base $\mathrm{D}$, throat diameter $\mathrm{d}$, angle of the conical section $\mathrm{C}$, length of the convex edges $\mathrm{B}$, and the anchor length $\mathrm{A}$, where the structure is attached to the wall of the cylindrical duct by their convex edges. Here, the cylindrical duct is a microfluidic tube resembles to a blood vein.

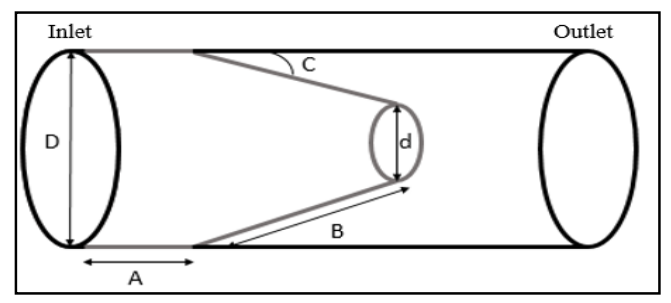

Fig.1. 2D schematic representation of a biomimetic microvalve.

\subsection{The microvalves mechanism}

In nature, venous valves are present to prevent a retrograde flow of biological fluid such as blood. Generally, they consist of a pair of intimal folds attached to the wall of the vein (Fig.2). These folds or leaflets are usually crescent-shaped with free edges extending in the direction of blood flow (Hossler F.E et al. 1988). Venous valves allow blood flow in its natural course toward the heart while restricting the reverse flow by bringing the opposed edges into contact (Qing Yu et al. 2001).

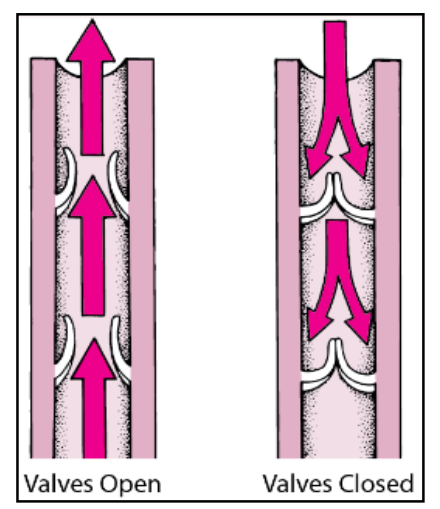

Fig.2. Venous valves (James D. Douketis, 2019). 
The objective of this study is to create soft biomimetic microvalves for the design of novel structure with the same mechanism as that of the venous valves. The key principle of this microvalve is the fluid flow through the cylindrical conduct and especially the flexibility of the material that our microvalves are made of. Subsequently, it is evident that the fluid displacement within the microvalve must occur in the situation of throat closing and opening. A schematic presentation of the function of our biomimetic microstructure is depicted in Fig.3.a, b.

The inlet flow rate $\mathrm{Q}_{+}$is larger than the outlet flow rate $\mathrm{Q}_{-}$as the flow resistance in diverging direction is smaller than that in converging direction at small diverging angles. When we applied the pressure gradient in inlet of the cylindrical duct, we obtained the movement of the liquid from the base to the throat (positive direction) (Fig.3.a), which causes an enlargement of the throat of the microvalves. Moreover, this enlargement favoured the passage of the liquid. Conversely, when the pressure was applied in the opposite direction, we obtained the movement of the liquid from the throat to the base (negative direction) (Fig.3.b); this induced a narrowing in the throat, which prevented the passage of the liquid.

On the other hand, the selection of the material is very important. Here, the microvalves were formed from Polymer materials. In our study, we used Poly Di Methyl Siloxane (PDMS). Therefore, the polymer materials provide many advantages regarding cost, mechanical proprieties, and ease of processing. PDMS was particularly selected for its elastic properties, knowing that its Poisson's ratio is close to 0.49. In addition, this material is ranked with biocompatible materials and considered to be the most suitable and adaptable material for flexibility in fabrication, such as soft lithography. The flexible, soft and highly elastic nature allows good sealing of a microfluidic system and minimises fluidic leakage. The transparent material also eased optical detection of flow in our microvalves.

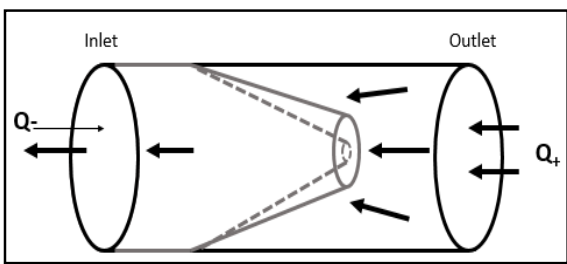

(a)

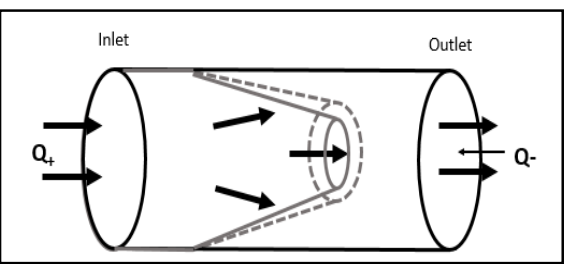

(b)

Fig.3. The schematic view of Operational principle; (a): positive direction. (b): negative direction.

\subsection{Theorical analysis of $3 D$ microvalves}

The microvalve is analysed quantitatively by implementing a finite element model with the Comsol Multiphysics software COMSOL Multiphysics User's Guide (2012), which is capable of performing fluid flow analysis, solid mechanics analysis, and coupling between both these analyses. The brief description about the background of numerical modelling, geometric details of the microvalve model and boundary loading conditions are given in this section.

The Fluid-Structure Interaction interface combines fluid flow with structural mechanics, using a moving mesh to capture the fluid movement. The interface provides a predefined interface condition for the solid-fluid boundary. In the FSI approach, the fluid flow described using Navier-Stokes equations is combined with the solid mechanics at the interface of FSI boundaries. The physics interface uses an Arbitrary Lagrangian-Eulerian (ALE) method to combine the fluid flow formulated using an Eulerian description and a spatial frame (where the material moves through the computational domain) with solid mechanics formulated using a Lagrangian description and a material frame (where the computational domain moves with the material. 
(Feldt C et al. 2002, Y. Xu et al. 2014). Regarding the theoretical concepts, the fluid flow in the channel is described by the Navier-Stokes equations, solving for the velocity field $u_{-}$fluid $=(\mathrm{u}, \mathrm{v})$ and the pressure $\mathrm{p}$. The Navier Stokes equations that govern the fluid motion are given by:

$$
\begin{gathered}
\rho\left(u_{\text {fluid }} \cdot \nabla\right) u_{\text {fluid }}=\nabla[-p I+K]+F \\
\rho \nabla \cdot u_{\text {fluid }}=0 \\
K=\mu\left(\nabla u_{\text {fluid }}+\left(\nabla u_{\text {fluid }}\right)^{T}\right)
\end{gathered}
$$

In Eq. (1), $\rho$ is the fluid density ( $\rho$ water $=1010 \mathrm{~kg} / \mathrm{m} 3$ ), $u_{\text {fluid }}$ is the velocity of the fluid $p$ is the pressure, $\mathrm{I}$ is the unit diagonal matrix and $\mathrm{F}$ is the volume force affecting the fluid. We assume no gravitation or other volume forces affecting the fluid, so $\mathrm{F}=0$.

To solve Eq. (1), we need to impose the boundaries conditions on the fluid domain: Гin Гin U Гwall U Гout :

$$
\begin{array}{rll}
n^{T}[-p L+K] . n=-p_{0} & \text { on } & \text { Tin } \\
{[-p L+K] . n=-p_{0} . n} & \text { on } & \text { Tout } \\
u_{\text {fluid }}=0 \text { on } & \text { Гwall } &
\end{array}
$$

At the inlet boundary, it was imposed a pressure value $\operatorname{Tin} \Gamma_{\text {in }}$. It must be noted that $u_{\text {fluid }} . t=0, p_{0} \geq p_{0}$ where $n$ is the unit vector exterior to Гin Eq. (4).

At the outlet boundary Гout, it must be noted that $p_{0} \leq p_{0}$ and $n$ is the outward unit normal to Гout Eq. (5).

On the wall Гwall, a no-slip boundary condition Eq. (6), was imposed.

The structural deformations are solved for using an elastic formulation and a nonlinear geometry formulation to allow large deformations.

The equation of motion for solid mechanics part is obtained using the first Piola-Kirchhoff stress tensor as:

$$
0=\nabla \cdot(F S)^{T}+F_{V}
$$

In Eq. (7), the tensor divergence operator $\nabla$ computed with respect to coordinates of material frame, where strain-displacement relationship matrix $F$ is given as follows:

$$
F=I+\nabla u_{\text {solid }}
$$

In Eq. (9), $S$ is the second Piola-Kirchoff stress and $j$ is the Jacobian determinant.

$$
S=S_{a d}+j_{i} F_{\text {inel }}^{-1}\left(C: \varepsilon_{\text {ei }}\right) F_{\text {inel }}^{-T}
$$

Where:

$$
j=\operatorname{det}(F)
$$




$$
\begin{gathered}
\varepsilon_{e l}=\frac{1}{2}\left(F_{e l}^{T} F_{e l}-1\right) \\
F_{e l}=F F_{\text {inel }}^{-1} \\
S_{a d}=S_{0}+S_{e x t}+S_{q} \\
\varepsilon=\frac{1}{2}\left[\left(\nabla u_{\text {solid }}\right)^{T}+\nabla u_{\text {solid }}+\left(\nabla u_{\text {solid }}\right)^{T} \nabla u_{\text {solid }}\right] \\
C=C(E . \vartheta)
\end{gathered}
$$

The spatial frame also deforms with a mesh deformation that is equal to the displacements $\mathrm{u}$ of the solid within the solid domains. The mesh is free to move inside the fluid domains and it adjusts to the motion of the solid walls. This geometric change of the fluid domain is automatically accounted for in Comsol Multiphysics by the ALE method.

The Boundary condition of the PDMS microvalve is fixed at its edges and considered a zero displacement boundary condition, as demonstrated in Eq (16).

$$
u_{\text {solid }}=0
$$

As already mentioned, the microvalves walls were modelled as a linear elastic material, the boundary conditions for fluid model are no-slip at the fluid-wall interface. FSI and pressure are defined at both inlet and outlet of the microvalves (Fig.4). The fluid is considered incompressible; moreover, the flow in a continuously deforming geometry is solved using the ALE technique.

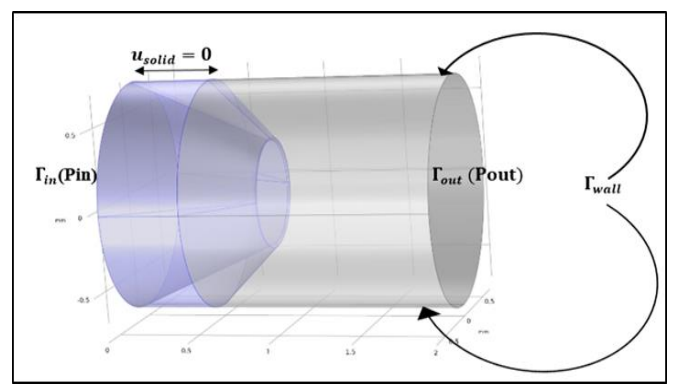

Fig. 4. Representation of the computational domain; microvalve geometry constructed on Comsol Multiphysics.

\section{Results and discussions}

We studied the fluid flow rate based on several parameters in the aim of gathering all the criteria that meet our work. Accordingly, the changing of the geometric parameters and the applied pressure can also affect the fluid flow rate. The geometric parameters were taken as A, B, D and angle of the conical section $\mathrm{C}$ (cited above).

To study the effect of pressure variation on the flow rate; we compare the various parameters of the microvalves according to different direction (forward direction and backward direction). Therefore, to determinate the efficiency of the microvalve, we must study the behaviour of the fluid in both directions. From these characteristics, we can determine an efficiency: 


$$
E_{f f}=\frac{Q_{p}-Q_{r}}{Q_{p}}
$$

Where $Q_{p}$ and $Q_{r}$ are the flow rates, for identical pressure conditions $P$, in the forward direction and the resistant direction, respectively. The most important role of this parameter is the determination of the optimal geometric structure, which allows eliminating the maximum of the return of the flow.

Figures 5, 6, 7, 8 and 9 show the graphs of fluid flow $(\mathrm{ml} / \mathrm{min})$ according to the pressure (Pa) fluctuation when fluid flows through microvalves with different geometries. We applied several pressure values at the system inlet, and for each analysis, it is desired to keep only one geometry parameter changing while the other geometry parameters remain constant. The given pressures are in increasing order. On the other side, it is noteworthy that the flow rate in the forward direction (desired way) is greater in relation to the backward direction (undesired way); this remark depends on the efficiency relationship.

In this part, we studied the fluctuation of the flow as a function of the pressure by varying several parameters. First, we started by the effect of divers anchor length A of the microvalves. We compared the flow rate to different directions depending on the length A. Figure 5 shows the flow rate-pressure curves to different anchor length $(\mathrm{A} 1=0.9 \mathrm{~mm}, \mathrm{~A} 2=0.5 \mathrm{~mm}, \mathrm{~A} 3=0.2 \mathrm{~mm})$ where the other parameters are considered constant. It can be seen that the flow rate increases with the increase in pressure. Furthermore, the decrease of the anchor length maximises the fluid pumped, which also maximises the flow rate of the fluid. This phenomenon is justified by flexibility of the microvalve. As a result, it makes the structure more mobile; it opens and closes more freely. However, there is a proportionality of efficiency in flow rate between passing (desired way) and resistant direction (undesired way).

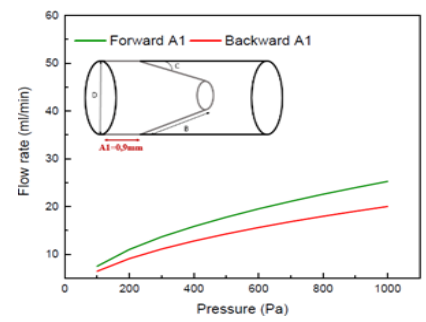

(a)

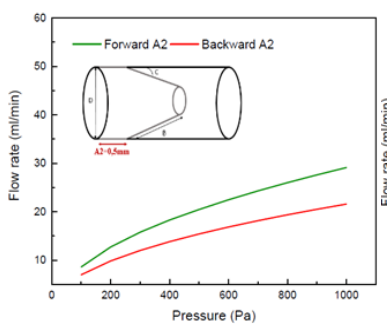

(b)

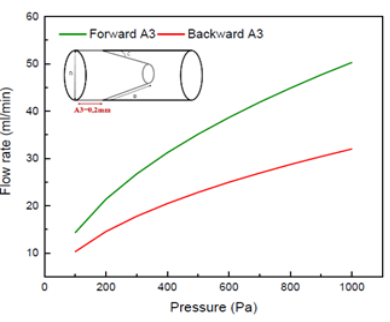

(c)

Fig.5. Curves between pressure and flow rate of different anchor length A. (a): A1 $=0.9 \mathrm{~mm}$, (b): $\mathrm{A} 2=0.5 \mathrm{~mm},(\mathrm{c}): \mathrm{A} 3=0.2 \mathrm{~mm}$.

The curves in Fig.6 show the flow rate-pressure curves to different convex edges length B (B1=0.5 mm, B2=1.2 mm, B3=1.8 mm). As shown in the curves, when $B 1=0.5 \mathrm{~mm}$, the micropump flow rate decreases to the minimum $\mathrm{Q}=58 \mathrm{ml} / \mathrm{min}$ with an efficiency from $57 \%$ between the forward and backward direction. Also, when B2 $=1.2 \mathrm{~mm}$, the flow rate continues its evolution, but the gap between the flow rate in the positive and negative direction decreases to $34 \%$. However, when $\mathrm{B} 3=1.8 \mathrm{~mm}$, there is a re-increase at $44 \%$ and the flow rate reaches the maximum $\mathrm{Q}=92 \mathrm{ml} / \mathrm{min}$. Thus, we can say that the relation flow rate-length is directly proportional. 


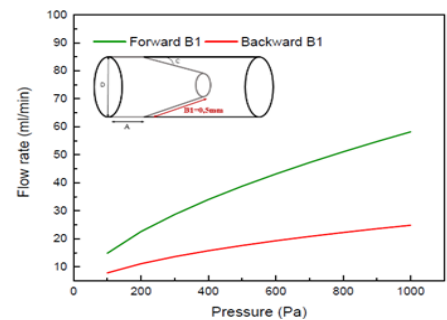

(a)

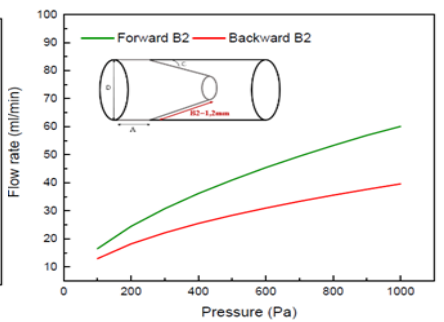

(b)

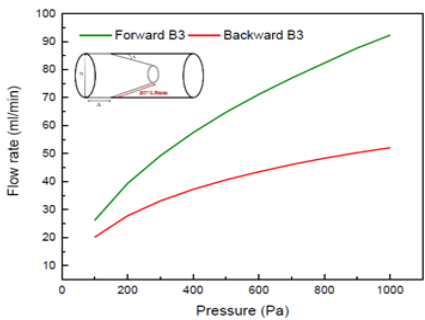

(c)

Fig.6. Curves between pressure and flow rate of different convex edges length B. (a): $\mathrm{B} 1=0.5 \mathrm{~mm},(\mathrm{~b}): \mathrm{B} 2=1.2 \mathrm{~mm},(\mathrm{c}): \mathrm{B} 3=1.8 \mathrm{~mm}$.

Figure 7 shows the flow rate-pressure curves to different conical section angle $\mathrm{C}$. It can be seen from the figure that the flow rate of the microvalve is maximum when $\mathrm{C} 3=50^{\circ}$, followed by $\mathrm{C} 2=60^{\circ}$, and minimum when $\mathrm{C} 1=70^{\circ}$. The reason is that when $\mathrm{C} 1=70^{\circ}$ and the channel length $\mathrm{B}$ is relatively long, few fluids can pass through the inlet and outlet caused by the narrow and spaceoccupied channel, resulting in a small flow rate and slow acceleration with the increasing pressure. In addition, the flow in both directions is almost equal, which implies a very low efficiency of the microvalve; on the other hand, when $\mathrm{C} 2=60^{\circ}$ the flow rate is greater than the former because of the wider micro channel. However, it is too narrow and weakens the microvalve function of the conical angle. The flow difference through the inlet and outlet does not effectively increase and the steady flow is not large enough. When $\mathrm{C} 3=50^{\circ}$, the microvalve function adequately achieves the largest flow rate among the three with increased efficiency between the forward and resistive direction. However, beyond this angle we lose the efficiency and form of our conical shape.

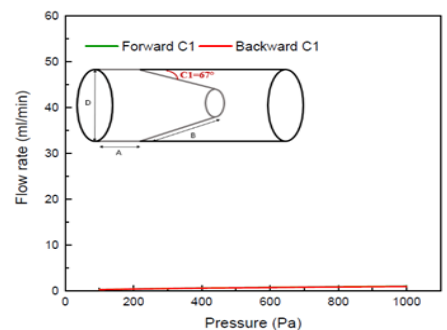

(a)

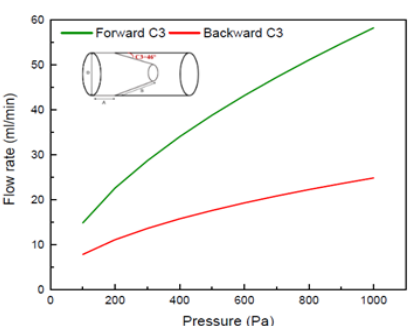

(b)

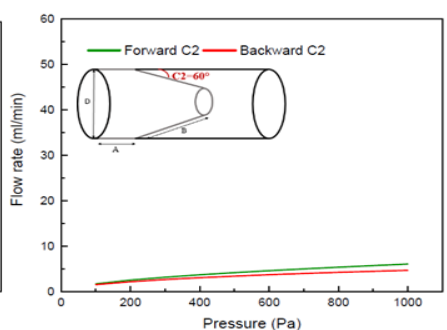

(c)

Fig.7. Curves between pressure and flow rate of different angle C. (a): $\mathrm{C} 1=67.24^{\circ}$, (b):

$$
\mathrm{C} 2=60.83^{\circ},(\mathrm{c}): \mathrm{C} 3=46.16^{\circ}
$$

In this part, we studied the behaviour of the fluid according to the opening of the base. Fig. 8 shows the flow rate-pressure curves to different diameters taken at the base. Thus, the flow rate is proportional to the applied pressure. As it can be seen from the figure; when the opening is large, favours more passage of the fluid, which implies an increase in the volume of the fluid. When $\mathrm{D} 3=1.8 \mathrm{~mm}$, the microvalve generates the maximum flow rate $\mathrm{Q}=60 \mathrm{ml} / \mathrm{min}$. In the same way, we obtain an increase in efficiency between the forward and backward direction from $3 \%$ to $61 \%$ in D1=1.2 $\mathrm{mm}$ and D3=1.8 $\mathrm{mm}$, successively. 


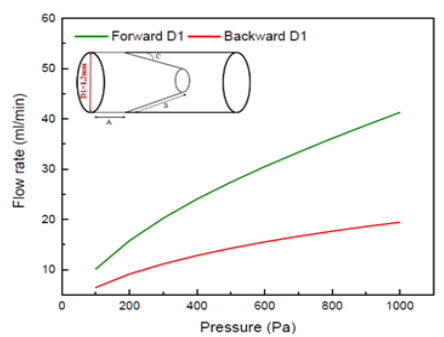

(a)

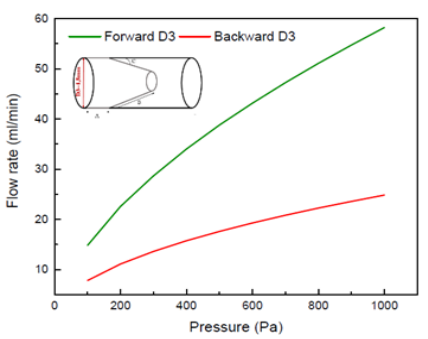

(b)

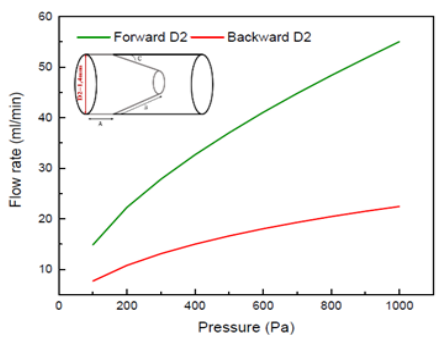

(c)

Fig.8. Curves between pressure and flow rate of different diameter of the base D. (a): $\mathrm{D} 1=1.2 \mathrm{~mm}$, (b): D2=1.4mm, (c): D3=1.6mm.

Another interesting point is that as Young's modulus of PDMS, knowing that the modulus of elasticity is influenced by degree of crosslinking between base polymer and curing agent, this value increases with increase in base polymer.

In our study, we chose three values of Young's modulus while varying the dosage of the crosslinking agent. Figure 9 shows the flow rate-pressure curves according to different Young's modulus; for E1=375 MPa, E2=750 MPa and E3=1500 MPa with two orders of magnitude between the chosen values. From this figure, we can deduce that the flow rate increases with diminution of the Young's modulus, which is very logical. When the modulus is less important, the structure becomes more flexible, which favours the opening and closing of the microvalve.

Furthermore, the variation of this parameter allowed us to reduce the leakage of the microvalve while increasing the efficiency between the desired and undesired direction.

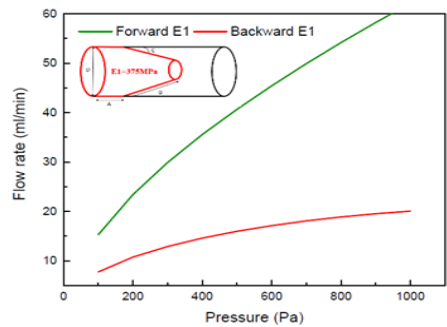

(a)

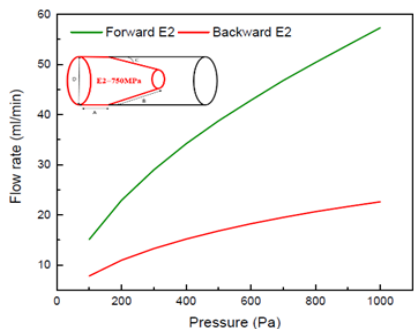

(b)

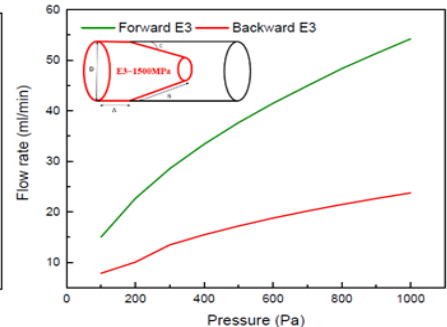

(c)

Fig.9 Curves between pressure and flow rate of different Young's modulus. (a): E1=375MPa, (b): $\mathrm{E} 2=750 \mathrm{MPa},(\mathrm{c}): \mathrm{E} 3=1500 \mathrm{MPa}$

Based on these results, we have been able to understand the governing factors, which are associated with our microvalve. Therefore, it is useful to study the microvalve performance for various parameters and conclude how structure's dimensions affect the flow rate. Therefore, not only the volume flow is taken into consideration, but we also focus on the efficiency of the microvalve. Therefore, by analysing the results in the forward and backward direction, a best design is to be selected to implement in the microvalve.

The performance of various models were analysed by plotting various geometry parameters against the quality parameter $E_{f f}$ as shown in previous figures. In our analysis, the value of $E_{f f}$ was calculated as described earlier in Eq. (17). 
Figure 10a is a plot of A against $E_{f f}$, keeping B, D and C constant. There is no specific trend shown by this plot for a given pressure. Thus, there is little variation of $E_{f f}$, with geometry parameter A and changing of the gap of nearly $8 \%$ between each efficiency at given anchor length. Hence, it is not an important parameter for the microvalve geometry.

In addition, we studied the variation of $\mathrm{D}$ against $E_{f f}$ keeping $\mathrm{A}, \mathrm{B}$, and $\mathrm{C}$ constant as shown in Fig.10b. This graph shows a very small variation in Eff with increase in $D$. In $D=0.8 \mathrm{~mm}$ we had an efficiency of the order of $39 \%$ to increase at $53 \%$ in $\mathrm{D}=1.2 \mathrm{~mm}$, while in $\mathrm{D}=1.4 \mathrm{~mm}, 1.6$ $\mathrm{mm}$, and $1.8 \mathrm{~mm}$ there was no specific trend and we had just a $2 \%$ gap.

Figure 10c represents the plot of B against $E_{f f}$ keeping A, D, and C constant. It is evident from the figure that the value of Eff first decreases from $57 \%$ to $44 \%$ and then continues to go down slowly with a $1 \%$ gap. This curve shows a very small variation in $E_{f f}$ with an increase in B.

Afterwards, Fig.10d shows the plot of E against $E_{f f}$ keeping A, B, C and D constant. It is evident that the efficiency decreases slowly with the increase in the modulus of elasticity. According to these results, the structure becomes more rigid. Which prevents the opening and closing of the microvalve and, consequently, there will be more leakage between the passing and resisting direction. We had a value of efficiency located in the range between $60 \%$ and $80 \%$ for the minimum and the maximum of Young's modulus, respectively.

Finally, Fig.10e indicates the variation angle C according to $E_{f f}$ keeping A, B and D constant. From this curve, we can deduce that the maximum value of Eff decreased by the increasing angle of the conical section with an important gap from each angle. It starts from $8 \%$ and continues to $65 \%$. That is to say, at the beginning, we have the flow in the passing direction almost equal to the resistant direction, and then it continues to improve by changing the angle in order to minimize the reflux.

It is also clear that the variation of $E_{f f}$ with geometry parameter A, B and D is very small compared with the angle of conical section C. Hence, angle $\mathrm{C}$ is the key parameter of the microvalve characteristics.

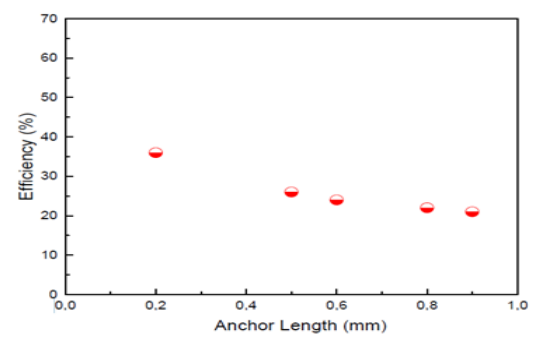

(a)

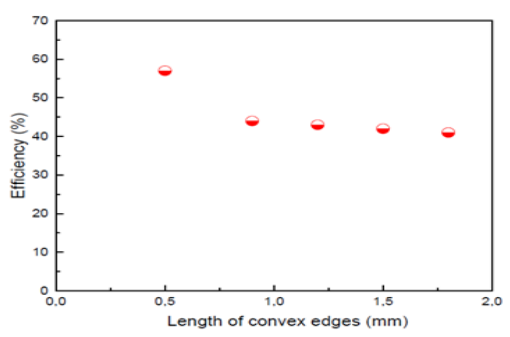

(c)

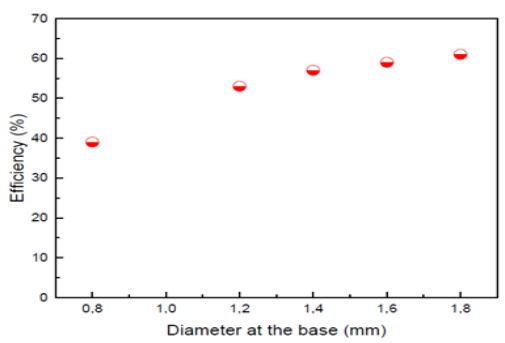

(b)

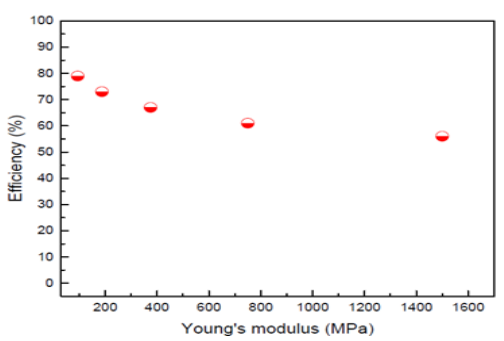

(d) 


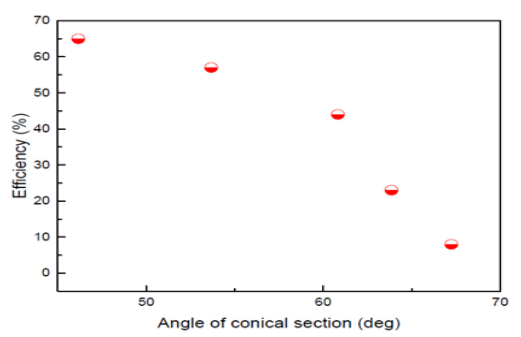

(e)

Fig.10. (a): microvalve efficiency as a function of anchor length A, (b): microvalve efficiency as a function of diameter at the base $\mathrm{D}$, (c): microvalve efficiency as a function of length of the convex edges B, (d): microvalve efficiency as a function of Young's modulus, (e): microvalve efficiency as a function of conical angle $\mathrm{C}$.

Considering the constructed microvalve model, two studies were done. Phase A: with boundary conditions where the fluid flows through the microvalve in the positive direction (forward direction). Phase B: with boundary conditions where the fluid flows through the microvalve in the negative direction (backward direction). In order to make a prediction of the behaviour of the microvalve qualitatively, we studied the effect of pressure variation on our structure deformation; for this purpose, we considered the displacement parameter to determine how much the microvalve opens and closes.

(a)

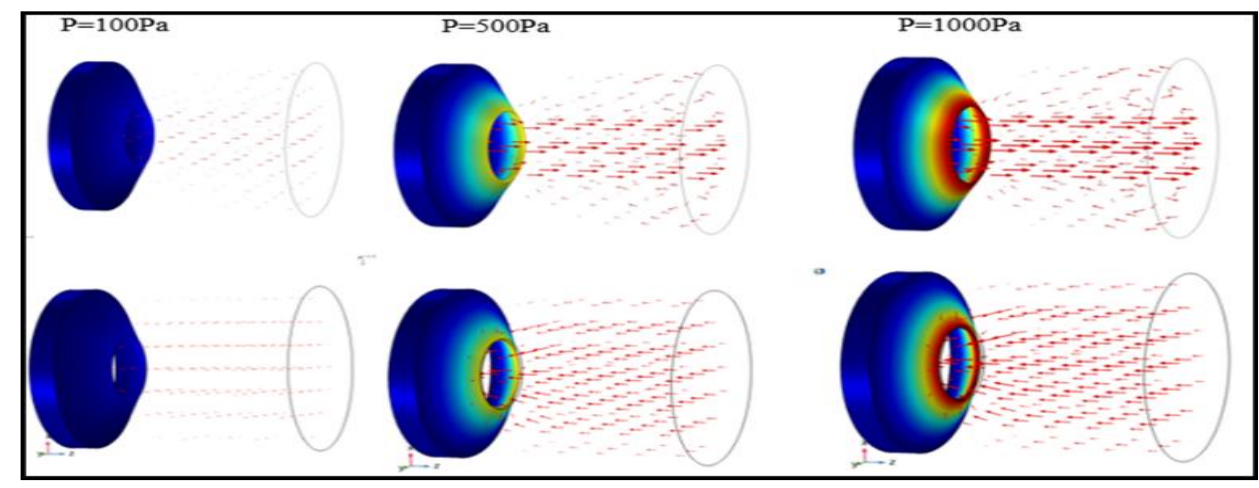

Fig.11. Microvalve model; snapshots of the displacement parameter and flow patterns (with the red streamlines) in (a): forward direction, (b): backward direction.

Figure $11 \mathrm{a}, \mathrm{b}$ describes the respective microvalve behaviour. In the first phase, the microvalve model stays in its initial state with a slight displacement at $\mathrm{P}=100 \mathrm{~Pa}$. From this pressure, the deformation phase is verified. In $\mathrm{P}=500 \mathrm{~Pa}$, the throat opens with a displacement higher than the previous one with a greater order of magnitude, and increases with a doubled value for $\mathrm{P}=1000 \mathrm{~Pa}$, when the throat is remarkably open (Fig.11a). In the second phase, the behaviour reverses; the passage of the fluid in the negative direction causes the closing of the throat while increasing the applied pressure. In $\mathrm{P}=100,500$ and $1000 \mathrm{~Pa}$, the displacement is increased with an order of magnitude at each pressure value applied (Fig.11b). The microvalve opens and closes due to the gradient of pressure imposed across the microvalve that contributes to the increase of the flow. 
The objective of this demonstration is not to reach the maximum opening of the microvalve's throat but to show its flexibility and ability to deform while applying the gradient of pressure.

\section{Conclusion}

The new generation of microvalves was designed and modelled qualitatively by implementing a finite element model using Comsol Multiphysics software. In this paper, we have established 3D biomimetic microvalves based on a cone-shaped structure in which the fluid flow cross through the cylindrical duct. It is evident that the fluid displacement within the microvalve and flexibility of the structure must occur by opening and closing of the throat. The microvalve geometry effect was studied by calculating the forward and reverse direction of the flow for different pressures. Efficiency parameter $E_{f f}$ was used to compare the various geometries studied. It was observed that the angle of conical section is the key parameter for the performance of this type of microvalve compared with other parameters. The current study was focusing on the effect of the geometric parameters on the flow rate. From these results, we deduce that our new microvalve can be incorporated in micro devices for medical application.

\section{NOMENCLATURE}

$\begin{array}{ll}\text { A } & \text { anchor length } \\ \mathrm{B} & \text { length of the convex edges } \\ \mathrm{C} & \text { angle of conical section } \\ \mathrm{D} & \text { diameter at the base } \\ \mathrm{E} & \text { Young's modulus } \\ \mathrm{E}_{\mathrm{ff}} & \text { efficiency } \\ \mathrm{F} & \text { volume force } \\ \mathrm{I} & \text { unit diagonal matrix } \\ J & \text { deformation Jacobian } \\ n & \text { unit vector exterior in } \Gamma_{\text {in }} \\ n & \text { outward unit normal in } \Gamma_{\text {out }}\end{array}$

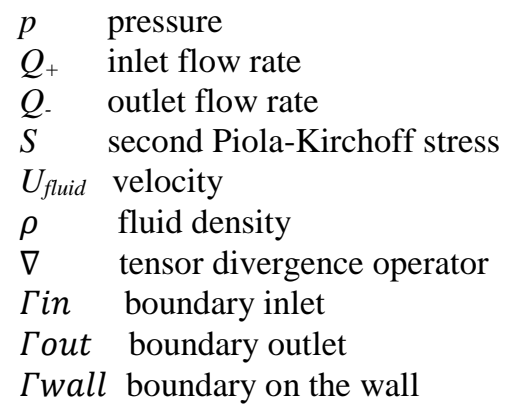

\section{REFERENCES}

Amrani, I.; A. Cheriet and M. Feliachi. (2018). Design and experimental investigation of a bidirectional valveless electromagnetic micro-pump. Sensors and Actuators A: Physical, 272, 310-317.

Aleksandr K. (2017). Numerical model of the pneumatic diaphragm actuator. Mechanics and Advanced Technologies. doi.org/10.20535/25211943.2017.81120008.

Barkat, T, F. Z. Kadid, M. S. Aggoune, R. Abdessemed. (2018). Modeling and Simulation of a Circular Valveless Micropump. Journal of the Serbian Society for Computational Mechanics. DOI: 10.24874/jsscm.2018.12.02.07.

Cui, Q.; Liu, C.; and Zha, X.F.W. (2006). Design and simulation of a piezoelectrically actuated micropump for the drug delivery system. Proceedings of the IEEE International Conference on Automation Science and Engineering. Shanghai, China, 45-50.

COMSOL Multiphysics User's Guide, Protected by U.S. Patents 7,519,518; 7,596,474; and 7,623,991. Patents pending, (May 2013).

Feldt. C, L. Chew. (2002). Geometry-based macro-tool evaluation of non-moving-part valvular microchannels. J. Micromech. Microeng. 12 (2002) 662-669. 
Huang, Y.-F., C.-H Tsou, C.J .Hsu, Y.C Lin, T. Ono, Y.C. Tsai. (2020). Metallic glass thin film integrated with flexible membrane for electromagnetic micropump application. Japanese Journal of Applied Physics, 59(SI), SIIK03. doi:10.35848/1347-4065/ab82a7.

He, X., J. Zhu, X. Zhang. L. Xu, Song Yang. (29 September 2015). The analysis of internal transient flow and the performance of valveless piezoelectric micropumps with planar diffuser/nozzles elements. Microsyst Technol. doi : 10.1007/s00542-015-2695-0.

He, X, Zhuo. H, Deng. X, Wang.J, Li. F. (2011). The Optimization Analysis on Piezoelectric Micromixer With Modified Tesla Tubes. ASME-JSME-KSME 2011 Joint Fluids Engineering Conference. Hamamatsu, Shizuoka, JAPAN.

Hossler, F.E, R.F. West. (1988). Venous valve anatomy and morphometry: Studies on the duckling ascular corrosion casting. American Journal of Anatomy, 181(4), 425-432,

James D. Douketis, MD, McMaster University. Oct 2019 <https://www.msdmanuals.com/home/heart-and-blood-vessel-disorders/venous disorders/overview-of-the-venous-system>

Jin-yuan. Q, C. Hou, L. Xiao-juan, J. Zhi-jiang. (2020). Actuation Mechanism of Microvalves: A Review. Micromachines, 11, 172; doi: 10.3390/mi11020172.

Kang, J. and G. W. Auner. (2011). Simulation and verification of a piezoelectrically actuated diaphragm for check valve micropump design. Sensors and Actuators A: Physical, 167(2), 512-516. doi:10.1016/j.sna.2011.01.012.

Kang, J, J.V. Mantese, G.W. Auner. (2008). A self-priming, high performance, check valve diaphragm micropump made from SOI wafers. Journal of Micromechanics and Microengineering. http://iopscience.iop.org/0960-1317/18/12/125021.

Kwang. W.O, H. A. Chong. January (2006). A review of microvalves, J. Micromech. Microeng. R13-R39, 5 doi:10.1088/0960-1317/16/5/R01.

Liang-Yin. C, W. Wang. (2017). Microfluidic Fabrication of Microvalve-in-a-Chip. Microfluidics for Advanced Functional Polymeric Materials, 267-293.ch13. doi:10.1002/9783527803637.

Lefevre. R., A. Salette., C Agraffeil, J. Guillen, C. P. Déhan, L. Montès. (2012). Numerical study of bimetallic actuated micro-membrane with large deformations. Journal of Micromechanics and Microengineering, 23(1), 015011. doi:10.1088/0960-1317/23/1/015011.

Laser. D.J, J.G. Santiago. (2004) A review of micropumps. Journal of Micromechanics and Microengineering. vol.14, pp.35-64, doi: 10.1088/0960-1317/14/6/R01.

Marwan, N, M. A. Mohamed Sultan, R. Tariq, M. Kamyar. (2019). Geometrical Analysis of Diffuser-Nozzle Elements for Valveless Micropumps. 6th International Conference on Smart Instrumentation, Measurement and Applications (ICSIMA 2019). Kuala Lumpur, Malaysia.

McAllister, D.V.; Allen, M.G.; and Prausnitz, M.R. (2000). Microfabricated microneedles for gene and drug delivery. Annual Review of Biomedical Engineering, 2, 289-313.

Nordina, A.N, A. A. Zainuddina, Ab. R. Rosminazuin, I. Voiculescub, W. Cheung Makc, (2017). Screen Printed Electromechanical Micro-Total Analysis System ( $\mu$ tas) For Sensitive and Rapid Detection of Infectious Diseases. Procedia Technology. doi: 10.1016/j.protcy.2017.04.043.

Nabel K. A. (2016). Diaphragm Actuator Design with New Rubber Compounds. al-muthanna journal for engineering sciences. DOI: 10.18081/mjet/2016-4/81-87.

Nickolay Lavrik. V., J. Michael Sepaniak, G. Panos Datskos. (2004). Cantilever transducers as a platform for chemical and biological sensors. Review of Scientific Instruments, 75(7), 22292253. doi:10.1063/1.1763252.

Partha K. D, A. B. M. T Hasan. (2017). Mechanical micropumps and their applications. 7th BSME International Conference on Thermal Engineering. Dhaka, Bangladesh.8. doi: 10.1063/1.4984739. 
Ramchandani. P, J. Daudpoto, M.A. unar. (2017). Shape Memory Alloy Actuated Inchworm. Engeneering Science And Technology Internarional Research JournalL, VOL.1, NO.4.

Srinivasa. C. R. (2020). a numerical study on thermal stress analysis of a micro electro mechanical systems (mems) bimetallic actuator. International Journal of Mechanical and Production Engineering Research and Development (IJMPERD). DOI: 10.24247/ijmperdjun202029.

Shrikant K. Y, S. B. Rupesh. (2019). Shape Memory Alloy Actuators: A Review. International Journal for Research in Applied Science \& Engineering Technology (IJRASET). doi: 10.22214/ijraset.2019.5134.

Saggere, L. (2015). Membrane Actuation for Micropumps. Encyclopedia of Microfluidics and Nanofluidics, 1741-1746. doi:10.1007/978-1-4614-5491-5_871

Victor. S, R. Christian , B. Christoph , Marko Klaus Baller. Microvalve. Patent: US8753587B2. (june2014).

Wenpeng Liu, H. Zhang, H. Zhao, Z. Tang, Y. Wang, C. Sun, Wei. P, X. Duan. (2017). Comparative analysis of static and non-static assays for biochemical sensing using on-chip integrated field effect transistors and solidly mounted resonators. Sensors and Actuators B: Chemical, 243, 775-783. doi:10.1016/j.snb.2016.12.065

Wang. X. Y, Y.T. Ma, G. Y. Yan, Z. H. Feng. (2014). A compact and high flow-rate piezoelectric micropump with a folded vibrator. Smart Materials and Structures. vol 23, doi:10.1088/0964-1726/23/11/115005.

Woias. P. (2005). Micropumps-past, progress and future prospects. Sensors and Actuators B.vol.105, pp. 28-38, doi:10.1016/j.snb.2004.02.033.

Xu, Y, W. Yan, T. Cao, L. Guo. (June 18-20, 2014). Study on the Valveless Micropumps with Saw-tooth Microchannels. Proceedings 11th IEEE International Conference on Control \& Automation (ICCA), Taichung, Taiwan,

Yang, C.H., Y.L. Hsieh, P.H. Tsou, B. R. Li, (2019). Thermopneumatic suction integrated microfluidic blood analysis system. PLOS ONE, 14(3), e0208676. doi:10.1371/journal.pone.0208676.

Yu, Q, J. M. Bauer, J. S Moore, D. J. Beebe. (2001). Responsive biomimetic hydrogel valve for microfluidics. Applied Physics Letters, 78(17), 2589-2591. doi:10.1063/1.1367010.

Yu, Q, J. M. Bauer, J. S Moore, D. J. Beebe, (12-14 Oct. 2000). Fabrication and charaterization of a biomimetic hydrogel check valve. Annual International IEEE-EMBS Special Topic Conference on Microtech. Lyon, France.

Zolotov, Y. A. (2020). Evolution of chemical analysis methods. Herald of the Russian Academy of Sciences, 90(1), 56-62. doi:10.1134/s1019331620010220.

Zhang, J. H., Y. Wang, J. Huang. (27 April 2017). Advances in Valveless Piezoelectric Pump with Cone-shaped Tubes. Chinese Journal of Mechanical Engineering, 30(4), 766-781. doi: 10.1007/s10033-017-0151-z. 